\title{
Rezensionen online 705
}

\section{... von der Redaktion betreut (Juli - September 2016)}

Die Redaktion der Vierteljahrshefte für Zeitgeschichte arbeitet seit $2003 \mathrm{im}$ Auftrag des Instituts für Zeitgeschichte München - Berlin mit dem Rezensionsjournal sehepunkte zusammen. Diese Kooperation findet nicht nur in den sehepunkten ihren Niederschlag, sondern auch in den Vierteljahrsheften selbst: In jedem Heft werden die von der Redaktion angeregten und betreuten Rezensionen angezeigt, die in den drei Monaten zuvor in den sehepunkten erschienen sind.

Martin Böhm, Die Royal Air Force und der Luftkrieg 1922-1945. Personelle, kognitive und konzeptionelle Kontinuitäten und Entwicklungen, Paderborn 2015. Rezensiert von: Bernd Lemke (Zentrum für Militärgeschichte und Sozialwissenschaften der Bundeswehr, Potsdam) in sehepunkte 16 (2016), Nr. 9

http:/ / www.sehepunkte.de/2016/09/28108.html

Micha Brumlik, Wann, wenn nicht jetzt? Versuch über die Gegenwart des Judentums, Berlin 2015.

Rezensiert von: Moshe Zimmermann (The Hebrew University of Jerusalem) in sehepunkte 16 (2016), Nr. 9

http://www.sehepunkte.de/2016/09/28674.html

António Costa Pinto / Aristotle Kallis (eds.), Rethinking Fascism and Dictatorship in Europe, Basingstoke 2014.

Rezensiert von: Grzegorz Rossolinski-Liebe (Friedrich-Meinecke-Institut, Freie Universität Berlin) in sehepunkte 16 (2016), Nr. 7/8

http://www.sehepunkte.de/2016/07/27062.html

Tiziana Di Maio, Alcide De Gasperi und Konrad Adenauer. Zwischen Überwindung der Vergangenheit und europäischem Integrationsprozess (1945-1954), Frankfurt/M. [u.a.] 2014.

Rezensiert von: Holger Berwinkel (Berlin) in sehepunkte 16 (2016), Nr. 9 http://www.sehepunkte.de/2016/09/26849.html

Gregor Feindt, Auf der Suche nach politischer Gemeinschaft. Oppositionelles Denken zur Nation im ostmitteleuropäischen Samizdat 1976-1992, Berlin / Boston 2015. Rezensiert von: Florian Peters (Institut für Zeitgeschichte München - Berlin) in sehepunkte 16 (2016), Nr. $7 / 8$

http://www.sehepunkte.de/2016/07/28246.html

Regina Fritz / Éva Kovács / Béla Rásky (Hgg.), Als der Holocaust noch keinen Namen hatte. Zur frühen Aufarbeitung des NS-Massenmordes an den Jüdinnen und Juden, Wien 2016.

Rezensiert von: Katarzyna Person (Jüdisches Historisches Institut, Warschau) in sehepunkte 16 (2016), Nr. 9

http:/ / www.sehepunkte.de/2016/09/28979.html 
Sarah Gensburger, Witnessing the Robbing of the Jews. A Photographic Album, Paris, 1940-1944, Bloomington / Indianapolis, IN 2015.

Rezensiert von: Christoph Kreutzmüller (Jüdisches Museum, Berlin) in sehepunkte 16 (2016), Nr. $7 / 8$

http:/ / www.sehepunkte.de/2016/07/28956.html

Kirsten Gerland, Politische Jugend im Umbruch von 1988/89. Generationelle Dynamik in der DDR und der Volksrepublik Polen, Göttingen 2016.

Rezensiert von: Rainer Eckert (Berlin) in sehepunkte 16 (2016), Nr. 9

http:/ / www.sehepunkte.de/2016/09/29008.html

Brigitte Halbmayr, Herbert Steiner. Auf vielen Wegen, über Grenzen hinweg. Eine politische Biografie, Weitra 2015.

Rezensiert von: Ina Markova (Wien) in sehepunkte 16 (2016), Nr. 7/8

http:/ / www.sehepunkte.de/2016/07/28777.html

Hans-Christian Harten, Himmlers Lehrer. Die Weltanschauliche Schulung in der SS 1933-1945, Paderborn 2014.

Rezensiert von: Niels Weise (Institut für Zeitgeschichte München - Berlin) in sehepunkte 16 (2016), Nr. 9

http:/ / www.sehepunkte.de/2016/09/26715.html

Lothar Höbelt (Hg.), Aufstieg und Fall des VdU. Briefe und Protokolle aus privaten Nachlässen 1948-1955, Wien u. a. 2015.

Rezensiert von: Margit Reiter (Zentrum für Holocaust-Studien am Institut für Zeitgeschichte München - Berlin) in sehepunkte 16 (2016), Nr. 7/8

http://www.sehepunkte.de/2016/07/28563.html

Georg Hoffmann, Fliegerlynchjustiz. Gewalt gegen abgeschossene alliierte Flugzeugbesatzungen 1943-1945, Paderborn 2015.

Rezensiert von: Susanne Meinl (Lollar) in sehepunkte 16 (2016), Nr. 9

http:/ / www.sehepunkte.de/2016/09/28120.html

Barbara J. Keys, Reclaiming American Virtue. The Human Rights Revolution of the 1970s, Cambridge, MA 2014.

Rezensiert von: Robert Brier (London) in sehepunkte 16 (2016), Nr. 9

http:/ / www.sehepunkte.de/2016/09/26736.html

Stephan Kieninger, Dynamic Détente. The United States and Europe, 1964-1975, Lanham, MD 2016.

Rezensiert von: Rolf Steininger (Innsbruck) in sehepunkte 16 (2016), Nr. 9

http://www.sehepunkte.de/2016/09/28657.html

Johannes Koll, Arthur Seyß-Inquart und die deutsche Besatzungspolitik in den Niederlanden (1940-1945), Wien u. a. 2015. 
Rezensiert von: Maya I.S. Gradenwitz (Goethe-Universität, Frankfurt/M.) in sehepunkte 16 (2016), Nr. $7 / 8$

http://www.sehepunkte.de/2016/07/27787.html

Franziska Kuschel, Schwarzhörer, Schwarzseher und heimliche Leser. Die DDR und die Westmedien, Göttingen 2016.

Rezensiert von: Alina Laura Tiews (Forschungsstelle Mediengeschichte, Hamburg) in sehepunkte 16 (2016), Nr. 9

http://www.sehepunkte.de/2016/09/28688.html

David Landau, Arik. The Life of Ariel Sharon, Or Yehuda 2015.

Rezensiert von: Tamar Amar-Dahl (Berlin) in sehepunkte 16 (2016), Nr. 7/8

http://www.sehepunkte.de/2016/07/29141.html

Linke Betriebsintervention, wilde Streiks und operaistische Politik 1968 bis 1988. Arbeit - Bewegung - Geschichte. Zeitschrift für historische Studien 2016/I, Berlin 2016.

Rezensiert von: Jens Benicke (Freiburg/Brsg.) in sehepunkte 16 (2016), Nr. 9

http://www.sehepunkte.de/2016/09/29337.html

Simo Mikkonen / Pia Koivunen (eds.), Beyond the Divide. Entangled Histories of Cold War Europe, New York / Oxford 2015.

Rezensiert von: Claudia Kemper (Hamburger Institut für Sozialforschung / Universität zu Köln) in sehepunkte 16 (2016), Nr. 9

http:/ / www.sehepunkte.de/2016/09/27907.html

Ministère des Affaires Etrangères, Documents diplomatiques français 1971. Tome I (1er janvier - 30 juin), Bruxelles [u.a.] 2015.

Rezensiert von: Ulrich Lappenküper (Otto-von-Bismarck-Stiftung, Friedrichsruh) in sehepunkte 16 (2016), Nr. 7/8

http:/ / www.sehepunkte.de/2016/07/28158.html

Ministère des Affaires Etrangères, Documents diplomatiques français 1971. Tome II (1er juillet - 31 décembre), Bruxelles [u.a.] 2015.

Rezensiert von: Ulrich Lappenküper (Otto-von-Bismarck-Stiftung, Friedrichsruh) in sehepunkte 16 (2016), Nr. 7/8

http://www.sehepunkte.de/2016/07/28158.html

Peter Polak-Springer, Recovered Territory. A German-Polish Conflict over Land and Culture, 1919-1989, New York / Oxford 2015.

Rezensiert von: Jim Bjork (King's College London) in sehepunkte 16 (2016), Nr. 7/8

http://www.sehepunkte.de/2016/07/28309.html

Peter M. Quadflieg, Gerhard Graf von Schwerin (1899-1980). Wehrmachtgeneral, Kanzlerberater, Lobbyist, Paderborn 2015. 
Rezensiert von: Reinfried Brunsch (Freising) in sehepunkte 16 (2016), Nr. 9 http:/ / www.sehepunkte.de/2016/09/27524.html

Srinath Raghavan, 1971. A Global History of the Creation of Bangladesh, Cambridge, MA 2013.

Rezensiert von: Amit Das Gupta (Universität der Bundeswehr München) in sehepunkte 16 (2016), Nr. $7 / 8$

http:/ / www.sehepunkte.de/2016/07/25516.html

Sebastian Rick, Die Entwicklung der SED-Diktatur auf dem Lande. Die Landkreise Liebenwerda und Schweinitz in der Sowjetischen Besatzungszone 19451949, Göttingen 2016.

Rezensiert von: Jens Schöne (Landesbeauftragter für die Unterlagen des Staatssicherheitsdienstes der ehemaligen DDR, Berlin) in sehepunkte 16 (2016), Nr. 7/8

http:/ / www.sehepunkte.de/2016/07/28193.html

Thomas Riegler, Im Fadenkreuz. Österreich und der Nahostterrorismus 1973 bis 1985, Göttingen 2011.

Rezensiert von: Gisela Diewald-Kerkmann (Universität Bielefeld) in sehepunkte 16 (2016), Nr. 9

http:/ / www.sehepunkte.de/2016/09/20725.html

Michael Schüring, „Bekennen gegen den Atomstaat“. Die evangelischen Kirchen in der Bundesrepublik Deutschland und die Konflikte um die Atomenergie 19701990, Göttingen 2015.

Rezensiert von: Kristian Buchna (Stiftung Bundespräsident-Theodor-Heuss-Haus, Stuttgart) in sehepunkte 16 (2016), Nr. 9

http:/ / www.sehepunkte.de/2016/09/27478.html

Rolf Steininger, Deutschland und der Nahe Osten. Von Kaiser Wilhelms Orientreise 1898 bis zur Gegenwart, Reinbek 2015.

Rezensiert von: Bettina Sophie Weißgerber (Ostfildern) in sehepunkte 16 (2016), Nr. 7/8

http:/ / www.sehepunkte.de/2016/07/27669.html

Corinna R. Unger, Entwicklungspfade in Indien. Eine internationale Geschichte 1947-1980, Göttingen 2015.

Rezensiert von: Amit Das Gupta (Universität der Bundeswehr München) in sehepunkte 16 (2016), Nr. 9

http:/ / www.sehepunkte.de/2016/09/27408.html

Anika Walke, Pioneers and Partisans. An Oral History of Nazi Genocide in Belorussia, Oxford / New York 2015.

Rezensiert von: Kerstin Baur (Institut für Zeitgeschichte München - Berlin) in sehepunkte 16 (2016), Nr. 9

http:/ / www.sehepunkte.de/2016/09/28445.html 
Gerhard Weigt, Demokratie jetzt. Der schwierige Weg zur deutschen Einheit. Ein Zeitzeuge berichtet, Leipzig 2015.

Rezensiert von: Bettina Tüffers (Kommission für Geschichte des Parlamentarismus und der politischen Parteien, Berlin) in sehepunkte 16 (2016), Nr. 9

http://www.sehepunkte.de/2016/09/28287.html

\section{Rezensionen zu Publikationen des IfZ (Juli - September 2016):}

Anselm Doering-Manteuffel / Lutz Raphael / Thomas Schlemmer (Hgg.), Vorgeschichte der Gegenwart. Dimensionen des Strukturbruchs nach dem Boom, Göttingen 2016.

Rezensiert von: Friederike Sattler (Goethe-Universität, Frankfurt/M.) in sehepunkte 16 (2016), Nr. 9

http://www.sehepunkte.de/2016/09/28321.html

Horst Möller u.a. (Hgg.), Die Einheit. Das Auswärtige Amt, das DDR-Außenministerium und der Zwei-plus-Vier-Prozess, Göttingen 2015.

Rezensiert von: Dieter H. Kollmer (Zentrum für Militärgeschichte und Sozialwissenschaften der Bundeswehr, Potsdam) in sehepunkte 16 (2016), Nr. 9

http:/ / www.sehepunkte.de/2016/09/28203.html

Andrea Bambi / Axel Drecoll (Hgg.), Alfred Flechtheim. Raubkunst und Restitution, Berlin / Boston 2015.

Rezensiert von: Tanja Bernsau (Institut für Kunstgeschichte, Justus-Liebig-Universität, Gießen) in sehepunkte 16 (2016), Nr. 9

http:/ / www.sehepunkte.de/2016/09/27848.html 


\section{Auswahl aus unseren Neuerscheinungen}

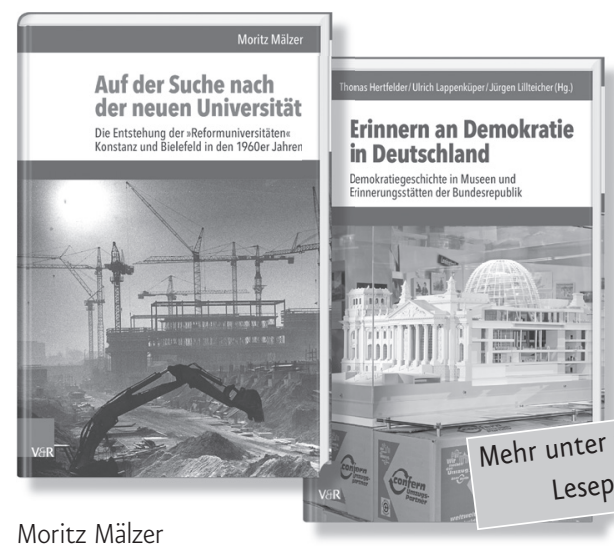

Auf der Suche nach der neuen Universität

Die Entstehung der "Reformuniversitäten"

Konstanz und Bielefeld in den 1960er Jahren

Bürgertum Neue Folge. Studien zur Zivilgesellschaft, Band 13. 2016. 512 Seiten mit 6 Abb., gebunden

$€ 80,-D$

ISBN 978-3-525-36852-7

Die Unis Konstanz und Bielefeld waren in den 1960er Jahren Prestigeprojekte der damaligen Hochschul- und Landespolitik.

Thomas Hertfelder / Ulrich Lappenküper /

Jürgen Lillteicher ( $\mathrm{Hg}$.)

\section{Erinnern an Demokratie in Deutschland}

Demokratiegeschichte in Museen und

Erinnerungsstätten der Bundesrepublik

2016. 344 Seiten mit 104 farbigen Abb., gebunden $€ 30,-D$

ISBN 978-3-525-30093-0

Warum spielt die Geschichte der Demokratie in der Erinnerungskultur Deutschlands nur eine geringe Rolle?



Verlagsgruppe Vandenhoeck $\because$ Ruprecht I Var unipress

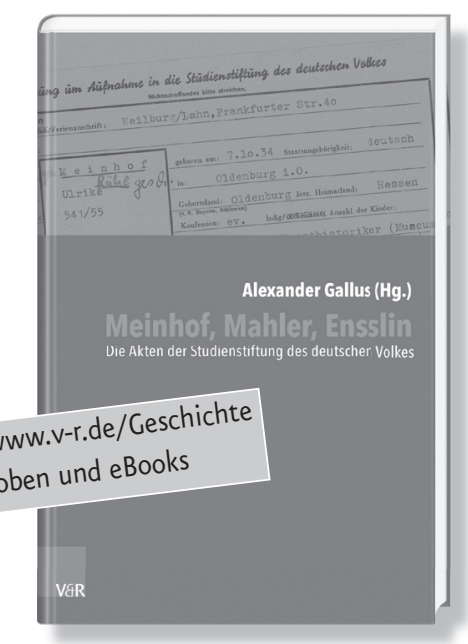

Alexander Gallus (Hg.)

\section{Meinhof, Mahler, Ensslin}

Die Akten der Studienstiftung des deutschen Volkes

Mit einem Vorwort des Präsidenten der Studienstiftung des deutschen Volkes Reinhard Zimmermann.

2016. 296 Seiten mit 16 Faksimiles, geb. $€ 60,-D$

ISBN 978-3-525-30039-8

Ulrike Meinhof, Horst Mahler und Gudrun Ensslin waren Mitbegründer der RAF. Sie alle gehörten dem exklusiven Stipendiatenkreis der Studienstiftung des deutschen Volkes an. Die Förderakten der drei befanden sich bislang unter Verschluss. Nun führt diese Dokumentation erstmals vor Augen, in welch beunruhigender und verstörender Weise auch Hochbegabung, Anerkennung und Förderung in Terrorismus und Gewalttätigkeit münden können.

www.v-r.de 